\title{
Targeted radionuclide therapy: frontiers in theranostics
}

\section{Francesca Gallivanone ${ }^{1}$, Mauro Valente ${ }^{2,3,4,5}$, Annarita Savi ${ }^{6}$, Carla Canevari ${ }^{6}$, Isabella Castiglioni ${ }^{1}$}

${ }^{1}$ Institute of Molecular Bioimaging and Physiology, National Research Council (IBFM-CNR), Milan, Italy, ${ }^{2}$ Instituto de Fisica E. Gaviola, CONICET, Argentina, ${ }^{3}$ Laboratory for Research and Instrumentation in Physics Applied to Medicine and X-Ray Imaging (LIIFAMIRX), National University of Cordoba, Cordoba, Argentina, ${ }^{4}$ Centro de Fisica e Ingenieriía en Medicina, CFIM, Universidad de La Frontera, Temuco, Chile, ${ }^{5}$ Departamento de Ciencias Fisicas, Universidad de La Frontera, Temuco, Chile, ${ }^{6}$ Department of Nuclear Medicine, Centre for Experimental Imaging, San Raffaele Scientific Institute, Milan, Italy

\section{TABLE OF CONTENTS}

1. Abstract

2. Introduction

3. Features of targeted radionuclide therapy

4. Role of Dosimetry in TRT - computational approaches and use of Monte Carlo simulations

5. Current clinical applications

5.1. Thyroid cancer

5.2. Neuroendocrine tumors

5.3. Hepatocellular carcinoma

5.4. Prostate cancer

\section{Conclusion}

7. References

\section{ABSTRACT}

The concept of targeted radionuclide therapy (TRT) relies on the use of injected nuclear medicine as treating agents, targeted at the cellular or molecular level. The growth of the interest in TRT was stimulated by the advances in radionuclide production and labeling as well as by the improvement in the knowledge of appropriate and specific molecular targets. In recent years, different studies on TRT were focused on the evaluation of radionuclide compounds able to combine imaging of the disease with TRT, in a theranostic approach. This approach is of particular interest towards the personalization of treatments, allowing both the baseline characterization of oncological pathologies and treatment optimization by correct dosimetric calculation as well as therapy monitoring. This paper presents a review of recent literature on TRT, with a particular focus on clinical applications promoting such a theranostic approach, showing the impact of the synergy of diagnostic imaging and therapeutics.

\section{INTRODUCTION}

Molecular Imaging (MI) techniques, such as Single Photon Emission computed Tomography and Positron Emission Tomography combined with Computed Tomography (SPECT/CT and PET/CT), have gained a central role for the diagnosis and therapy monitoring of oncological pathologies. The possibility to visualize in vivo, and non-invasively, biological processes at a molecular and cellular level allows oncological pathologies to be detected both at the baseline and during therapy, thus potentially enabling to personalize the diagnostic and therapeutic patient clinical work up. In particular, the increased sensitivity and image resolution of MI systems (e.g. three-dimensional acquisition (1), resolution recovery strategies in image reconstruction (2)) combined with the higher resolution of CT system, offered by current generation hybrid PET/CT systems, provide a better characterization of oncological lesions.

Even if, in clinical practice, image evaluation is often performed only by qualitative analysis, nuclear medicine techniques in oncology are inherently quantitative techniques measuring uptake of radiotracers in cancer cells. Many advances in processing methods have been applied to PET and SPECT images with the purpose to extract quantitative/semi-quantitative parameters from oncological lesions that can be used as in vivo biomarkers (3) for theranostics purposes. In order to increase the accuracy of quantitation, several strategies have been implemented to compensate 
for partial volume effect the measure of regional biomarkers in PET (4). More accurate quantitation leaded to the identification of accurate biomarkers for predicting therapy outcome (e.g. 5,6).

In recent years, a growing interest raised in the use of nuclear medicine compounds not only for qualitative and quantitative evaluation of oncological pathologies, but also as treating agents. The concept of targeted radionuclide therapy (TRT), using radionuclides for targeted therapy at the cellular or molecular level, is not new, but the availability of highly specific and selective radiopharmaceuticals together with the improvement in the knowledge of radiobiology promoted a larger diffusion of this therapeutical strategy in oncology. Different research studies were dedicated to this topic at different levels, from studies of basic science dedicated to evaluation of cells radiosensitivity or of chemical affinity of radionuclides and biological carrier, to pre-clinical studies were radiolabeled compound are tested in animal models and to clinical trials were the impact of TRT on patient outcome is evaluated.

Recent development in understanding of radionuclide chemistry and physical characteristics of radionuclides, as well as biological insights on appropriate and specific molecular targets allowed in recent years to combine imaging of the disease with radionuclide therapy. The theranostics approach of combining therapy and diagnostics is of particular interest towards the personalization of radionuclide therapy treatments. In this approach, diagnosis and therapy are combined using one single probe or using the same molecular target but with different radionuclides and imaging biomarkers may be used both to personalize the treatment as well as for dosimetric calculations before therapy and for therapy monitoring.

This paper presents a review of recent literature on TRT, with a particular focus on clinical applications promoting such an approach showing the impact of the synergy of combining diagnostic imaging and therapeutics. Particular attention will be given to the use of imaging biomarkers for dosimetric calculation, used for the tailoring of therapeutic treatment.

\section{FEATURES OF TARGETED RADIONUCLIDE THERAPY}

TRT is a systemic therapy using a radionuclide delivered to tumours through a radiopharmaceutical, which presents a high affinity to neoplastic cells, thus enabling killing tumor cells by the activation of the different pathways leading to DNA damaging. Even if both TRT and external radiotherapy (EBRT) use the same physical mechanism for cells killing (i.e. radiation-induced damage), profound differences exists from these therapeutical options. TRT allows a more continuous dose rate possessing a prolonged effect and high therapeutic index. EBRT alternates sessions with scheduled dose fractioning. Even if dose fractioning promotes mechanism of redistribution in cell cycle and re-oxygenation, inducing a radiosensibilization of tumor cells, the presence of sessions where no irradiation is performed thus allows the promotion of repair mechanisms and tumor cell repopulation. Unlike EBRT, ideally TRT should directly affect only cancer cells if appropriate targeting is performed, thus minimizing the irradiation of normal cells. Furthermore, TRT therapeutic efficiency is amplified following both effects related to physical characteristics of used radionuclides (e.g. cross fire effect) and to biological related reaction (e.g. bystander effect or cold damage mechanism related to targetcells interactions) (7). In TRT, radiation is directed to its target by a radiopharmaceutical with a specific binding to tumor tissue. This process is a highly dynamic process, both from a biochemical, biological and a physical point of view, causing a complex spatial and temporal distribution of the administered dose. This is a critical point both in terms of dose distribution, normal tissue damage and therapeutical efficacy (8). Advantages of TRT are related also to the possibility to treat multimetastatic disease in one shot.

A careful selection of the radionuclide, depending also to tumor biological features and characteristics, is required to ensure the efficacy of the TRT (9). Concerning the radionuclides, alpha and beta emitters as well as Auger electrons emitters are all suitable for TRT, but the choice of irradiation type should be tailored on the basis of tumor size and type. Small tumors or micrometastasis could benefit from the use of particle with a relatively short range. Cells with a known greater radiosensitivity could benefit by the use of emitting particles with high linear energy transfer and high energy. Physical half-life of radionuclides with respect to half-life clearance time of the pharmaceutical carrier should be accounted, considering both the in vivo pharmacokinetics of the radiolabeled compound, needed to localization into the target, and normal tissue irradiation. Important considerations have also to be performed regarding chemical aspects of the bounding with the carrier, in order to ensure the in vivo stability of the radiolabeled compound.

Furthermore, when TRT is accompanied by diagnostic imaging for dosimetry or therapy monitoring, gamma or $\mathrm{e}^{+}$emitters are required. This should be obtained in one single probe (e.g. ${ }^{131} \mathrm{I}$ ) or using two different radionuclides bound to the same pharmaceutical or, alternatively, designing specific therapy-diagnostic radiopharmaceutical pairs (10). In this view, carrier molecules should be designed to possess high affinity and specificity for the target and, if possible, a binding affinity to a variety of radionuclides. 
The main carriers used for TRT are antibodies and peptides, directed towards antigens selectively expressed by tumor cells, but also liposomes were investigated $(9,11)$.

As recognized by the joint workshops on TRT promoted by the Society of Nuclear Medicine, TRT was applied with significant clinical impact in thyroid cancer, lymphoma, prostate cancer, and neuroendocrine tumors even if current clinical trials are also addressed towards other malignancies (12-13).

\section{ROLE OF DOSIMETRY IN TRT - COMPUTATIONAL APPROACHES AND USE OF MONTE CARLO SIMULATIONS}

TRT relies on molecularly-driven delivery of radiation, thus requiring specific characterization and optimization. Radiation dosimetry in TRT is potentially useful for assessment and prediction of therapy efficacy and toxicity. Actually, patient-specific calculation of radiation doses delivered to tumors and normal tissues are routine in external beam radiotherapy (EBRT) and there exist great developments implementing both experimental and Monte Carlo based methods for EBRT. On the contrary, dosimetric applications for TRT have been not yet massive worldwide and radiation dosimetry for TRT, i.e. internal dosimetry, has not yet reached the state that dosimetry have in EBRT due to the complexity in quantification of dose distribution. In fact, most clinical studies have shown a relative low correlation between tumor dose and response and this is possibly due to the bystander effect and inaccuracies in dose estimation. Currently, the mechanisms associated with bystander and low-dose-rate effects are still under investigation, and questions remain on their impact in radionuclide therapy. In order to be optimal, patient-specific dosimetry in TRT, as well as in ERBT, requires anatomical images (CT or MR), as well as quantitative and kinetics estimation of radionuclide distribution using a nontherapeutic amount of the radiolabeled drug intended for subsequent therapy. This process is demanding in terms of human and methodological resources but it might be one of the keys for the optimization of TRT efficacy. The lack of knowledge of intrinsic characteristics of radiobiological effects as well as the difficulties in obtaining patientspecific biokinetics and imaging data is one of the major causes for preventing the spread of internal dosimetric methods for TRT, as standard routine in clinics (14).

Traditionally, planar imaging by means of gamma camera has been used to measure radioactivity inside the patient. Single Photon Emission computed Tomography (SPECT) and Positron Emission Tomography (PET) represent attractive methods to quantifying pharmacokinetics. Unfortunately, several factors contribute to the uncertainty in activity assessment, including photon scattering and attenuation, as well as limitations imposed by detector efficiency and image resolution. However, the introduction of hybrid modality, SPECT/ $\mathrm{CT}$ and $\mathrm{PET} / \mathrm{CT}$, provides data concerning the density of the body and photon transport allowing the introduction of corrections for attenuation and scattering. The limited spatial resolution, $(8-10 \mathrm{~mm}$ in SPECT and 5-6 $\mathrm{mm}$ in PET) resulted in large bias in the estimates of radioactivity concentration especially when imaging objects smaller 2-3 times the spatial resolution of the scanner, due to partial volume effect producing counting spill-over between different image regions because of the point spread function (PSF) of the system. In recent years, a great deal of work has gone into developing methods to compensate for the PSF both in SPECT and PET. Incorporation of PSF modelling in reconstruction algorithms (especially statistical methods) has been resulted in improvements in spatial resolution and noise (e.g. 15). Correction for PSF and partial volume effect is mandatory when voxel based dosimetry is used. Voxel dosimetry can be defined as the calculation of radiation absorbed at single voxel level (16). These methods calculate the absorbed dose to each voxel, usually by convolution of quantitative images of the activity distribution with a dose point kernel, which accounts for the spatially variant energy deposition resulting from the range of the irradiation particles. Voxel-based dosimetry enables approximate estimation of various dosimetric parameters, which include mean dose, maximum dose, minimum dose, dose uniformity, dose conformity, and dose volume histograms in the tumor, and surrounding organs. Subsequently, radiobiological models can be used to correlate these dosimetric parameters to clinical outcome, in terms of tumor control probability and normal tissue complication probability. Voxel-byvoxel correction strategies to recover the true activity distribution have been implemented for PET imaging, whereas for SPECT there are yet not well established procedures.

Treatment planning for molecular TRT can be strongly improved also by incorporating transportbased computational techniques, like the Monte Carlo (MC) techniques capable of calculating patient-specific dose distributions using a set of computed tomography scans to describe the 3D patient anatomy, combined with 2D (gamma camera planar images) and/or 3D (PET or SPECT) to describe the time-dependent radiation source (17-19). Different computational methods, based on actual patient anatomy and tissue composition as well as activity distributions, have been proposed with the aim of providing means for improving absorbed dose calculations in TRT applications. The objective consists in performing image fusion between patient-specific metabolic and anatomic data, thus attaining voxel-by-voxel representation of tissue and time-dependent activity. The emitted radiation, 
according to each radionuclide, is typically tracked in a voxel geometry defined from the morphological image acquired; whereas the assignment of specific tissue to each voxel is accomplished by means of dedicated CT calibrations providing tissue vs. Hounsfield index. Currently, the MC method is considered as the most accurate approach for the assessment of personalized dosimetry, demonstrating to be valid at human, tissue and cellular scales, based on the availability of imaging tools capable of accurately quantifying tissue density and activity distribution within the biological system (20-22).

In the case of clinical TRT, a distribution of radiolabeled compound is typically evaluated at different post-administration times, following the injection of a limited amount of radiopharmaceutical, with the aim of assessing time-activity curves (organ or voxel level), which are further used to infer the cumulated activity. This information, even if not exactly co-registered in practice, serves as the emission probability distribution required to define virtual voxel sources of radiation in the MC simulation where interactions between radionuclide emissions and the tissue are modelled taking into account all the different materials crossed by the particle along its track. The evaluation of the cumulated activity is then used to simulate dose release to both tumor and normal tissues in TRT. This approach can be also applied to evaluate the impact of absorbed dose, when a radiolabeled compound is used for diagnostic purpose. Moreover, performing MC calculations on complex systems allows obtaining reference data required as input for semi-analytic models, like dose point kernels (23-24) and S-values (25).

However, there are still some drawbacks to overcome. Although most of current MC codes accept the definition of volumes by means of "boundary representation", the tracking of the radioisotope emitted particles still may not be efficient enough $(16,20,23)$. Besides, each MC code has a definite maximum number of voxels that can be handled. As known, extremely fine sampling easily causes memory faults and it requires unreasonably long computational times. Moreover, $\mathrm{MC}$ as any other voxel-based computational method presents some practical limitations, because the requirement of a precise knowledge about cumulated activity demands sub-voxel precision in co-registration, which is not generally available. Furthermore, MC applications in TRT may require considerable computing resources, especially for extended geometries (whole body).

Advances for future implementation of $\mathrm{MC}$ techniques in routine clinics should be based on MC validation by means of different procedures including: different calculation approaches (i.e. different $\mathrm{MC}$ codes, different interaction models, different cross- sections, etc.), different patient representations and different geometry definitions (voxel-based vs. mathematical organ definition), among others. It might be expected that with increasing development in technology and computing science during the next decades, the MC method shall be the more suitable option for real-time calculations of radiation transport in patient-specific TRT applications.

\section{CURRENT CLINICAL APPLICATIONS}

\subsection{Thyroid cancer}

Differentiated thyroid cancer (DTC) is one of the first neoplasms where the concept of synergist approach of diagnostics and radionuclide therapy was applied. Radioiodine therapy was proven to be an effective treatment for DTC to selectively harm cells accumulating iodine (e.g. after surgery residual thyroid tissue as well as iodine-avid metastasis). $\beta$ emissions from ${ }^{131}$ I can be used for therapy while gamma rays, concomitantly emitted from the radionuclides, can be used to image cells accumulating the iodine. As mentioned above, pre- and/or peri- therapeutic ${ }^{131}$ | images performed using a gamma camera or a SPECT/CT scanner are strongly suggested in order to assess patient-specific absorbed dose and to tailor the therapeutical activity for the highest therapeutical efficacy without exceeding accepted limits for bone marrow and whole-body retention (26). Even if standard methodologies for calculation of absorbed dose using ${ }^{131}$ I diagnostic imaging were provided and advanced methods including also MC simulation were proposed (27), empirical evaluation of therapeutical ${ }^{131}$ | dose is performed in many nuclear medicine centers (28). This is mainly due to the simplicity and low costs of administering a fixed dose but could be explained also by the lack of studies showing significant impact of dosimetry in terms of the therapy outcome as well as by the debate of the impact of the diagnostic dose on the biokinetics of the therapeutical dose. In fact, since its first application, it was demonstrated an effect of reduction of therapeutic dose uptake of ${ }^{131}$ I, when a pretherapy diagnostic dose is administered for dosimetric purposes. This effect, called "stunning effect", was assumed to be dependent from the radiation damage due to the diagnostic tracer dose. In order to avoid the "stunning effect", scintigraphy using ${ }^{123}$ | was proposed for quantification of radioiodine uptake in the diagnostic phase, allowing for radiation dose planning optimization and for risk-benefit assessment with respect to other organ's irradiation. Physical characteristics of ${ }^{123}$ decay make this radioisotope a good candidate for imaging in scintigraphy (both in terms of gamma emission and in terms of count rate) with respect to ${ }^{131}$ I (29). Furthermore no "stunning effect" was shown for ${ }^{123}$, probably due to a low radiation burden with respect to ${ }^{131} \mid$ (29). Despite this its use for dosimetric assessment before radioiodine 
therapy is limited by a short half-life (13 h) with respect to ${ }^{131} \mathrm{I}$ and in its low sensitivity founded in particular for distant metastasis evaluation, preventing the complete assessment and definition of treatment planning (30).

${ }^{124} \mid-N a l$ was proposed as PET radiopharmaceutical to be used before treatment, for dosimetry and customization of radioiodine therapy in individual patients. In fact, ${ }^{124}$ I has a half-life $(4.2 . \mathrm{d})$ similar to that ${ }^{131}$ ( $\left.8.0 .2 \mathrm{~d}\right)$ and using PET imaging a superior lesion detectability is guaranteed with respect to scintigraphy or SPECT/CT. Pre-therapy ${ }^{124} \mathrm{l}-\mathrm{Nal}$ PET imaging was used for the estimation of dose for individual tumors subjected to subsequent radioiodine therapy. Capoccetti F et al. (2009) (31) performed individualized PET dosimetry in patients subjected to total thyroidectomy, which were referred to radioiodine therapy for ablation of thyroid remnant and treatment of metastasis. The authors conclude that ${ }^{124}$ I-Nal PET is an effective diagnostic tool for pre-therapy assessment of the disease spread and of individualized treatment planning. Furthermore they showed that no stunning effect was present and that the personalization of the treatment guaranteed an excellent percentage of success of remnants ablation.

Hobbs RF et al. (2009) (32) used ${ }^{124}$-Nal for patient-specific 3-dimensional radiobiologic dosimetry (3D-RD) in a pediatric case of metastatic papillary carcinoma after thyroidectomy and showed that 3D$\mathrm{RD}$ analysis is effective in providing details potential efficacy and toxicity of ${ }^{131} \mid$ treatment, highly impacting in particular in pediatric patients. Jentzen W. et al. (2008) (33) optimized a protocol with two point dosimetry for the evaluation of lesion dose per administered ${ }^{131}$ I activity of differentiated thyroid cancer metastases. Recently Jentzen W et. (2016) (34) used ${ }^{124}$ I-Nal PET imaging before and during initial radioiodine therapy, to evaluate treatment in patients with iodine-positive bone metastases, which results usually resistant to treatment with administration of a single standard dose. The pre-therapy PET data for dosimetry-guided ${ }^{131}$ I activity and for prediction of the average administered dose, while follow-up ${ }^{124}$ I-Nal PET were used to evaluate lesion-based therapy response. The authors found a high response rates corresponding to higher predicted administered doses, probably explaining the difficulty in achieving therapeutic efficacy for in bone metastasis when using a single standard dose.

\subsection{Neuroendocrine tumours}

Neuroendocrine tumors (NETs) are heterogeneous and rare neoplasms deriving from the neuroendocrine cell system with different localization and manifestation around the body, most frequently occurring in the gastrointestinal tract, pancreas and lungs. Biological studies have demonstrated that an overexpression of all five somatostatin receptors, in particular of SST2 receptors, is present in more than $80-90 \%$ of NETs, allowing these receptors as suitable targets for imaging and treatment. Radiolabeled somatostatin analogs were then developed for diagnostic imaging and also for peptide receptor radionuclide therapy (PRRT). Radioisotopes commonly used in PRRT are ${ }^{111} \mathrm{In},{ }^{90} \mathrm{Y},{ }^{177} \mathrm{Lu}$ and ${ }^{213} \mathrm{Bi}$. Different radiopharmaceuticals labeled with these radioisotopes were used and clinical validation and treatment effectiveness were evaluated (e.g. 35-36).

${ }^{111}$ In-DTPA-octreotide was the first radiopharmaceutical designed for PPRT in NETs. The advantage of using ${ }^{111}$ In regards the availability of both emissions, Auger-electron and gamma rays, for TRT and SPECT or SPECT/CT imaging, respectively; designing a theranostics compound, hence. Even if performing diagnostic imaging with this compound in accordance with the European Association for Nuclear Medicine guarantee an overall accuracy of $88 \%$ in lesion detection (37), its imaging potentials were not completely explored, both in terms of personalized pre-treatment dosimetry or therapy monitoring, no impacting tumor responses were measured, and the administered high doses of the compound resulted in bone marrow toxic effects (38-39).

Beta emitter ${ }^{90} \mathrm{Y}$ was then considered for labeling somatostatin analogues (e.g. (90Y-DOTA ${ }^{0}$, Tyr3)-octreotide). Different approaches were evaluated for individual dosimetry as well as for therapy monitoring (40). In a theranostics approach using the same probe both for imaging and therapy, the potential of the different emissions of ${ }^{90} \mathrm{Y}$ were evaluated. Since ${ }^{90} \mathrm{Y}$ has a low electron-positron pair emission $(0.0 .03 \%)$, studies were dedicated to use it as radioisotope for $\mathrm{PET}$ imaging, in order to measure absorbed doses after each cycle of PRRT and to optimize the activity injected in the following cycle (40). Also ${ }^{90} \mathrm{Y}$ bremsstrahlung SPECT images were considered for quantification of absorbed dose during therapy (41).

Despite the advantages of using the same radiopharmaceutical, in current clinical practice, the most used approach developed for dosimetry and monitoring purposes involves the use of surrogate for quantitative evaluation of a radiopharmaceutical compound. In this approach, an imageable counterpart of the therapy radiopharmaceutical is used for dosimetric simulations under the hypothesis that a similar pharmacokinetics is provided by similar physical and biological half-lives. This is the case, for example when using gamma rays of ${ }^{111} \mathrm{In}$, in the form of ${ }^{111} \mathrm{In}$-DOTATOC radiophamaceutical, for diagnosis, therapy monitoring and dosimetric evaluation and beta emissions of ${ }^{90} \mathrm{Y}$, using ${ }^{90} \mathrm{Y}$-DOTATOC radiophamaceutical, for therapeutical purposes. The effectiveness of this approach may be sometimes 
controversial due to structural changes that affect the receptor binding affinity (42).

${ }^{177} \mathrm{Lu}$ was evaluated for PRRT: patients treated with ( ${ }^{177}$ Lu-DOTA ${ }^{0}$, Tyr3)-octreotide (( $\left.{ }^{177} \mathrm{Lu}\right)$ DOTATATE) showed superior tumor response with respect to $\left({ }^{90} \mathrm{Y}\right)$ DOTATOC and fewer side effects. Furthermore, the advantages of using ${ }^{177} \mathrm{Lu}$ with respect to ${ }^{90} \mathrm{Y}$ rely on the fact that, besides beta emission, ${ }^{177} \mathrm{Lu}$ has also gamma emissions suitable for imaging purposes. Clinical quantification protocols of providing in vivo 3D dosimetry for ( $\left.{ }^{177} \mathrm{Lu}\right)$ DOTATATE PRRT using ${ }^{177} \mathrm{Lu}$ SPECT/CT images were validated (43).

Despite this, the high impact of $68 \mathrm{Ga}$ PET imaging on clinical management of patient with NET tumor (e.g. 44), due to the advantages of PET imaging vs SPECT images in terms of spatial resolution and quantification accuracy, stimulated the research on the use of approaches using PET imaging with ${ }^{68} \mathrm{Ga}$ (e.g. Standardized Uptake Value SUV or Ki), as surrogate methodology for treatment planning and therapy monitoring in ${ }^{177}$ Lu-PRRT (45-46).

\subsection{Hepatocellular carcinoma}

Hepatocellular carcinoma (HCC) is a common cause of worldwide mortality. Transarterial chemoembolization (TACE) is one of the standard locoregional treatments for $\mathrm{HCC}$, whereas transarterial radioembolization (TARE) using $\beta$-emitting ${ }^{90} Y$ has been developed as an alternative to TACE in recent years. Transarterial radioembolization (TARE) is a catheterbased procedure allowing delivering microspheres loaded with a radioactive isotope directly to the tumor using vascular systems supporting the tumor. Even if in this kind of radionuclide therapy there is not a biological entity as target for radiopharmaceutical, the target can be defined as the neoangiogenesis characterizing tumors. Microspheres, often loaded by ${ }^{90} \mathrm{Y}$, travel through the vascular bed to the smallest arterial branches that supply blood to the neoplastic lesion and they remain trapped. Here, the radioactive isotope radiates selectively neoplastic mass, giving the highest dose to the tumor while preserving normal tissues. TARE is indicated for patients with unresectable, intermediate stage $\mathrm{HCC}$ and, according to available clinical studies, it represents a promising therapy treatment for primary hepatocellular carcinoma (HCC) or for liver metastasis (47).

TARE is an example of a therapeutical approach which necessarily requires the use of an associated imaging, in order to ensure the procedure to be carried out safely, performing correct treatment planning and monitoring.

From a technical point of view, patients eligible for TARE perform a diagnostic angiography
(48). Angiographic imaging using a catheter allows showing vascular anatomy and portal vein patency as well as evaluating embolization of the collateral vessels. From the same catheter, macroaggregates of albumin (MAA) labeled by ${ }^{99 \mathrm{~m} T c}$ are injected, which have diffusion in vascular systems, similar to that of microspheres. Using ${ }^{99 \mathrm{~m} T C}$ SPECT/CT, it is then possible to simulate and predict the distribution of the microspheres into the liver. This pre-treatment imaging procedure is of great impact both on the evaluation of presence of activity outside the liver and into the lung, avoiding the consensus to perform the treatment, and on the treatment planning and personalization.

Different interesting studies were dedicated to evaluation and quantification of pre- and post- therapy imaging in TARE. Lam MG et al, (2013) developed a dual tracer ${ }^{99 \mathrm{~m} T C-M M A} /{ }^{99 \mathrm{~m}} \mathrm{TC}-\mathrm{SC}$ fusion SPECT strategy as a physiology-based method for improving personalized activity planning (49). Smits MLJ et al. explored the performance of ${ }^{166} \mathrm{Ho}$-microspheres. These spheres are an alternative to ${ }^{90} Y$ that can be imaged during treatment both using SPECT through ${ }^{166} \mathrm{Ho}$ gamma emission, and MR using paramagnetic properties of holmium. The authors showed that the advantages of using multimodal imaging allows in vivo dosimetry during treatment ensuring personalization of treatment as well as evaluation of inadequately treated tumors (50).

\subsection{Prostate cancer}

The use of TRT in prostate cancer ( $\mathrm{PCa}$ ) was promoted by the biological studies on prostate-specific membrane antigen (PSMA), which is a transmembrane protein expressed in all types of prostatic tissue that was found overexpressed in $90-100 \%$ of prostate cancer lesions as well as in lymph nodes and metastasis from PCa. PSMA was then evaluated as target of interest for imaging and therapy in PCa. Different antiPSMA probes, labeled with different radioisotopes, were developed both for imaging and radionuclide therapy, showing from moderate to high affinity for PSMA and anti-PSMA radiopharmacutical entered in routinary clinical diagnostic and therapeutical workup (51). In recent years, research efforts were dedicated to the evaluation of theranostics probe allowing both diagnosis and therapy in PCa. Weineisen $\mathrm{M}$ et al (2015) (52) proposed a $\left({ }^{68} \mathrm{Ga} /{ }^{177} \mathrm{Lu}\right) \mathrm{PSMA}-\mathrm{I} \& \mathrm{~T}$ compound that was optimized with respect to PSMA affinity and in vivo stability and that was tested in patients. $\left({ }^{68} \mathrm{Ga}\right)$ PSMA-I\&T used as PET radiotracer showed an efficient detection of metastatic PCa and his therapeutical counterpart, the ( $\left.{ }^{177} \mathrm{Lu}\right) \mathrm{PSMA}-\mathrm{I} \& \mathrm{~T}$, showed a promising treating efficiency both in clinical and in preclinical studies (52-53). Starting from these results, interesting in vitro and in vivo studies were carried out by Schottelius M et al (2015) using the same PSMA-I\&T compound, labeled with ${ }^{111}$ In to be imaged 
using SPECT/TC (53). This radiopharmaceutical was designed as complementary probe to $\left({ }^{68} \mathrm{Ga} /{ }^{177} \mathrm{Lu}\right)$ PSMA-I\&T for radio-guided surgery.

\section{CONCLUSIONS}

Significant efforts were dedicated to advances in TRT involving different disciplines starting from molecular and cell biology, towards nuclear and medical physics and chemistry. One of the paradigms that guide researches on this field is the possibility of exploit tumor-specific targeting agents with theranostic role, enabling both to extract imaging biomarkers for both disease characterization and monitoring and to be used for therapy. In this view different efforts were made at different levels, from the evaluation of molecular targets to the synthesis of radiolabeled compounds and its evaluation in preclinical models. As presented in this short review, TRT applications for different oncological pathologies were developed and used in a clinical context promoting such a synergic approach combining diagnostic imaging and therapeutics. The fully exploitation of this theranostic paradigm is required to clearly show the advantages of TRT for treatment of oncological pathologies, and for a personalization of patient clinical workup.

\section{REFERENCES}

1. V. Bettinardi, P. Mancosu, M. Danna, G. Giovacchini, C. Landoni, M. Picchio, MC. Gilardi, A. Savi, I. Castiglioni, M. Lecchi, F. Fazio: Two-dimensional vs threedimensional imaging in whole body oncologic PET/CT: a Discovery-STE phantom and patient study. Q J Nucl Med Mol Imaging 51(3), 214-23 (2007)

2. G. Rizzo, I. Castiglioni, G. Russo, MG. Tana, F. Dell'Acqua, MC. Gilardi, F. Fazio, S. Cerutti: Using deconvolution to improve PET spatial resolution in OSEM iterative reconstruction. Methods Inf Med 46(2), 231-5 (2007)

3. JW Prescott: Quantitative imaging biomarkers: the application of advanced image processing and analysis to clinical and preclinical decision making. J Digit Imaging 26(1), 97-108 (2013)

DOI: $10.1007 / \mathrm{s} 10278-012-9465-7$

4. F. Gallivanone, A. Stefano, E. Grosso, C. Canevari, L. Gianolli, C. Messa, MC. Gilardi, I. Castiglioni: PVE correction in PET-CT whole body oncological studies from PVEaffected images. IEEE Trans $\mathrm{Nucl} \mathrm{Sci}$ 58:736-747 (2011)

DOI: 10.1109/TNS.2011.2108316
5. F. Giganti, F. De Cobelli, C. Canevari, E. Orsenigo, F. Gallivanone, A. Esposito, I. Castiglioni, A. Ambrosi, L. Albarello, E. Mazza, L. Gianolli, C. Staudacher, A. Del Maschio: Response to chemotherapy in gastric adenocarcinoma with diffusionweighted MRI and (18) F-FDG-PET/CT: correlation of apparent diffusion coefficient and partial volume corrected standardized uptake value with histological tumor regression grade. J Magn Reson Imaging 40(5), 1147-1157 (2014)

DOI: 10.1002/jmri.24464

6. M. Picchio, M. Kirienko, P. Mapelli, I. Dell'Oca, E. Villa, F. Gallivanone, L. Gianolli, C. Messa, I. Castiglioni: Predictive value of pre-therapy (18)F-FDG PET/CT for the outcome of (18) F-FDG PET-guided radiotherapy in patients with head and neck cancer. Eur J Nucl Med Mol Imaging 41(1), 21-31 (2014)

DOI: $10.1007 / \mathrm{s} 00259-013-2528-2$

7. B. Blyth P. Sykes: Radiation-induced Bystander effects: what are they, and how relevant are they to human radiation exposures? Radiat Res 157, 139-57 (2011).

8. B. Brans, L. Bodei, F. Giammarile, O. Linden, M. Luster, WJ. Oyen, J. Tennvall. Clinical radionuclide therapy dosimetry: the quest for the "Holy Gray". Eur J Nucl Med Mol Imaging. 34(5):772-86 (2007).

DOI: $10.1007 / \mathrm{s} 00259-006-0338-5$

9. A. Dash, FF. Knapp, MR. Pillai MR. Targeted radionuclide therapy-an overview. Curr Radiopharm. 6(3):152-80 (2013). DOI: 10.2174/18744710113066660023

10. K. Zukotynski, H. Jadvar, J. Capala, F. Fahey: Targeted Radionuclide Therapy: Practical Applications and Future Prospects. Biomark Cancer 8(Suppl 2), 35-38 (2016) DOI: 10.4137/BIC.S31804

11. A. Aerts NR. Impens M. Gijs, M. D'Huyvetter, H. Vanmarcke, B. Ponsard, T. Lahoutte, A. Luxen, S. Baatout: Biological carrier molecules of radiopharmaceuticals for molecular cancer imaging and targeted cancer therapy. Curr Pharm Des 20(32), 5218-44 (2014) DOI: $10.2174 / 1381612819666140110114902$

12. F. Fahey, K. Zukotynski, J. Capala, N. Knight, Organizing Committee, Contributors, and Participants of NCl/SNMMI Joint Workshop on Targeted Radionuclide Therapy: Targeted radionuclide therapy: proceedings 
of a joint workshop hosted by the National Cancer Institute and the Society of Nuclear Medicine and Molecular Imaging. $\mathrm{J} \mathrm{NuCl}$ Med 55(2):337-48 (2014)

DOI: 10.2967/jnumed.113.135178

13. F. Fahey, K. Zukotynski, H. Jadvar, J. Capala, organizing committee, contributors, and participants of the second NCl-SNMMI Workshop on Targeted Radionuclide Therapy: Proceedings of the Second NCl-SNMMI Workshop on Targeted Radionuclide Therapy. J Nucl Med 56(7), 1119-1129 (2015).

DOI: 10.2967/jnumed.115.159038

14. G. Sgouros, SJ. Knox, MC. Joiner, WF. Morgan, Al. Kassis: MIRD continuing education: Bystander and low dose-rate effects: are these relevant to radionuclide therapy? J Nucl Med 48(10), 1683-91 (2007)

15. E. Rapisarda, V. Bettinardi, K. Thielemans, MC. Gilardi: Image-based point spread function implementation in a fully 3D OSEM reconstruction algorithm for PET. Phys Med Biol 55(14), 4131-51 (2010).

DOI: $10.1088 / 0031-9155 / 55 / 14 / 012$

16. Thomas SR: Options for radionuclide therapy: from fixed activity to patientspecific treatment planning. Cancer Biother Radiopharm 17(1),71-82 (2002)

DOI: $10.1089 / 10849780252824091$

17. C. Harfmann-Siantar, MA. Descalle, GL. DeNardo, DW. Nigg: Application of Monte Carlo Methods in Molecular Targeted Radionuclide Therapy. $12^{\text {th }}$ Biennial Topical Meeting of the Radiation Protection and Shielding Division of the American Nuclear Society, Santa Fe, USA, 2002.

18. P. Pérez, F. Botta, G. Pedroli, M. Valente. Dosimetry for beta-emitter radionuclides by means of Monte Carlo simulations. In: 12 Chapters on Nuclear Medicine, InTech, Chap. 11, 265-286, Ed: Dr. Ali Gholamrezanezhad (2011).

DOI: http://dx.doi.org/10.5772/25287

19. RL. Harrison, TK. Lewellen. The SimSET program. In: Monte Carlo Calculation in Nuclear Medicine: Applications in Diagnostic Imaging. Eds: M. Ljungberg, SE. Strand, MA. King (2012)

20. J. Seco, F. Verhaegen. Monte Carlo Techniques in Radiation Therapy. Eds: J. Seco, F. Verhaegen (2013).
21. H. Zaidi, XG. Xu: Computational Anthropomorphic Models of the Human Anatomy: The Path to Realistic Monte Carlo modeling in Radiological Sciences. Annu Rev Biomed Eng 9, 471-500 (2007)

DOI: 10.1146/annurev.bioeng.9.060906.151934

22. G. Flux, M. Bardies, M. Monsieurs, S. Savolainen, SE. Strands, M. Lassmann: EANM Dosimetry Committee. The impact of PET and SPECT on dosimetry for targeted radionuclide therapy. Z Med Phys 16(1), 47-59 (2006)

DOI: 10.1078/0939-3889-00291

23. F. Botta, A. Mairani, G. Battistoni, M. Cremonesi, A. Di Dia, A. Fassò, A. Ferrari, M. Ferrari, G. Paganelli, G. Pedroli, M. Valente: Calculation of electron and isotopes dose point kernels with fluka Monte Carlo code for dosimetry in nuclear medicine therapy. Med Phys 38(7), 3944-3954 (2011).

DOI: 10.1118/1.3586038

24. H. Uusijärvi, N. Chouin, P. Bernhardt, L. Ferrer, M. Bardiès, E. Forssell-Aronsson: Comparison of Electron Dose-Point Kernels in Water Generated by the Monte Carlo Codes, PENELOPE, GEANT4, MCNPX, and ETRAN. Cancer Bioth Radiopharm 24(4), 461-467 (2009)

DOI: $10.1089 / \mathrm{cbr} .2008 .0573$

25. N. Lanconelli, M. Pacilio, S. Lo Meo, F. Botta, A. Di Dia, LA. Torres Aroche, MA. Coca Pérez, M. Cremonesi: A free database of radionuclide voxel $S$ values for the dosimetry of nonuniform activity distributions. Phys Med Biol 57(1), 517-533 (2012)

DOI: $10.1088 / 0031-9155 / 57 / 2 / 517$

26. M. Lassmann, H. Hänscheid, C. Chiesa, C. Hindorf, G. Flux, M. Luster: EANM Dosimetry Committee series on standard operational procedures for pre-therapeutic dosimetry I: blood and bone marrow dosimetry in differentiated thyroid cancer therapy. Eur $\mathrm{J} \mathrm{Nucl} \mathrm{Med} \mathrm{Mol} \mathrm{Imaging} \mathrm{35,} \mathrm{1405-1412}$ (2008).

DOI: $10.1007 / \mathrm{s} 00259-008-0761-x$

27. M. Salvatori, M. Luster: Radioiodine therapy dosimetry in benign thyroid disease and differentiated thyroid carcinoma. Eur J Nucl Med Mol Imaging 37(4), 821-828 (2010) DOI: $10.1007 / \mathrm{s} 00259-010-1398-0$

28. BC. Ahn: Personalized Medicine Based on Theranostic Radioiodine Molecular Imaging 
for Differentiated Thyroid Cancer. Biomed Res Int 2016:1680464 (2016).

DOI: $10.1155 / 2016 / 1680464$

29. HM. Park. 123I: almost a designer radioiodine for thyroid scanning. $\mathrm{J} \mathrm{Nucl} \mathrm{Med}$ 43(1):77-8 (2002).

30. SD. Sarkar, TP. Kalapparambath, CJ. Palestro. Comparison of (123)I and (131)I for whole-body imaging in thyroid cancer. $J$ Nucl Med 43(5):632-4 (2002).

31. F. Capoccetti, B. Criscuoli, G. Rossi, F. Ferretti, C. Manni, E. Brianzoni. The effectiveness of 124I PET/CT in patients with differentiated thyroid cancer. Q J Nucl Med Mol Imaging 53(5):536-45 (2009).

32. RF. Hobbs, RL. Wahl, MA. Lodge, MS. Javadi, SY. Cho, DT. Chien, ME. Ewertz, CE. Esaias, PW. Ladenson, G. Sgouros. 124I PET-based 3D-RD dosimetry for a pediatric thyroid cancer patient: real-time treatment planning and methodologic comparison. $J$ Nucl Med 50(11):1844-7 (2009)

DOI: 10.2967/jnumed.109.066738

33. W. Jentzen, L. Freudenberg, EG. Eising, W. Sonnenschein, J. Knust, A. Bockisch. Optimized 124I PET dosimetry protocol for radioiodine therapy of differentiated thyroid cancer. J Nucl Med 49(6):1017-23 (2008).

DOI: 10.2967/jnumed.107.047159

34. W. Jentzen, F. Verschure, A. van Zon, R. van de Kolk, R. Wierts, J. Schmitz, A. Bockisch, I. Binse. 124I PET Assessment of Response of Bone Metastases to Initial Radioiodine Treatment of Differentiated Thyroid Cancer. J Nucl Med 57(10):1499-1504 (2016).

DOI: 10.2967/jnumed.115.170571

35. K. Oberg. Molecular Imaging Radiotherapy: Theranostics for Personalized Patient Management of Neuroendocrine Tumors (NETs). Theranostics,. 2(5):448-58 (2012). DOI: 10.7150/thno.3931

36. C. Kratochwil, FL. Giesel, F. Bruchertseifer, W. Mier, C. Apostolidis, R. Boll, K. Murphy, U. Haberkorn, A. Morgenstern. ${ }^{213} \mathrm{Bi}$-DOTATOC receptor-targeted alpha-radionuclide therapy induces remission in neuroendocrine tumours refractory to beta radiation: a first-in-human experience. Eur J Nucl Med Mol Imaging 41(11):2106-19 (2014).

DOI: 10.1007/s00259-014-2857-9
37. A. Pfeifer, U. Knigge, T. Binderup, J. Mortensen, P. Oturai, A. Loft, AK. Berthelsen, SW. Langer, P. Rasmussen, D. Elema, et al. 64CU-DOTATATE PET for Neuroendocrine Tumors: A Prospective Head-to-Head Comparison with 111In-DTPA-Octreotide in 112 Patients. J Nucl Med 56(6):847-54 (2015).

DOI: 10.2967/jnumed.115.156539

38. R. Valkema, M. De Jong, WH. Bakker, WA. Breeman, PP. Kooij, PJ. Lugtenburg, FH. De Jong, A. Christiansen, BL. Kam, WW. De Herder, et al. Phase I study of peptide receptor radionuclide therapy with [In-DTPA] octreotide: the Rotterdam experience. Semin Nucl Med 32:110-22 (2002).

39. LB. Anthony, EA. Woltering, GD. Espenan, MD. Cronin, TJ. Maloney, KE. McCarthy. Indium-111-pentetreotide prolongs survival in gastroenteropancreatic malignancies. Semin Nucl Med 32:123-32 (2002).

DOI: 10.1053/snuc.2002.31769

40. S. Walrand, GD. Flux, MW. Konijnenberg, R. Valkema, EP. Krenning, R. Lhommel, S. Pauwels, F. Jamar: Dosimetry of yttriumlabelled radiopharmaceuticals for internal therapy: $86 \mathrm{Y}$ or $90 \mathrm{Y}$ imaging? Eur J Nucl Med Mol Imaging 38(Suppl 1), S57-68 (2011)

DOI: 10.1007/s00259-011-1771-7

41. D. Minarik, K. Sjogreen Gleisner, $M$. Ljungberg: Evaluation of quantitative 90Y bremsstrahlung SPECT based on patient studies. J Nucl Med 50(2), 378 (2009).

DOI: 10.1088/0031-9155/53/20/008

42. JC. Reubi, JC. Schär, B. Waser, S. Wenger, A. Heppeler, JS. Schmitt, HR. Mäcke: Affinity profiles for human somatostatin receptor subtypes SST1-SST5 of somatostatin radiotracers selected for scintigraphic and radiotherapeutic use. Eur $\mathrm{J} \mathrm{Nucl} \mathrm{Med} \mathrm{27,}$ 273-282 (2000)

DOI: $10.1007 / \mathrm{s} 002590050034$

43. JC. Sanders, T. Kuwert, J. Hornegger, P. Ritt: Quantitative SPECT/CT Imaging of (177)Lu with In Vivo Validation in Patients Undergoing Peptide Receptor Radionuclide Therapy. Mol Imaging Biol 17(4), 585-593 (2015)

DOI: 10.1007/s11307-014-0806-4

44. A. Frilling, GC. Sotiropoulos, A. Radtke, M. Malago, A. Bockisch, H. Kuehl, J. Li, CE. Broelsch. The impact of $68 \mathrm{Ga}$-DOTATOC 
positron emission tomography/computed tomography on the multimodal management of patients with neuroendocrine tumors. Ann Surg 252(5):850-6 (2010).

DOI: 10.1097/SLA.0b013e3181fd37e8

45. I. Velikyan, A. Sundin, J. Sörensen, M. Lubberink, M. Sandström, U. GarskeRomán, H. Lundqvist, D. Granberg, B. Eriksson: Quantitative and qualitative intrapatient comparison of 68Ga-DOTATOC and 68Ga-DOTATATE: net uptake rate for accurate quantification. J Nucl Med 55(2), 204-210 (2014).

DOI: 10.2967/jnumed.113.126177

46. S. Ezziddin, J. Lohmar, CJ. Yong-Hing, A. Sabet, H. Ahmadzadehfar, G. Kukuk, HJ. Biersack, S. Guhlke, K. Reichmann: Does the pretherapeutic tumor SUV in $68 \mathrm{Ga}$ DOTATOC PET predict the absorbed dose of 177Lu octreotate? Clin Nucl Med 37(6), e141-147 (2012).

DOI: $10.1097 / R L U .0 b 013 e 31823926 e 5$

47. R. Sacco, C. Conte, E. Tumino, G. Parisi, S. Marceglia, S. Metrangolo, R. Eggenhoffner, G. Bresci, G. Cabibbo, L. Giacomelli: Transarterial radioembolization for hepatocellular carcinoma: a review. J Hepatocell Carcinoma 3:25-29 (2016)

DOI: 10.2147/JHC.S50359

48. F. Giammarile, L. Bodei, C. Chiesa, G. Flux, F. Forrer, F. Kraeber-Bodere, B. Brans, B. Lambert, M. Konijnenberg, F. BorsonChazot, J. Tennvall, M. Luster, the Therapy, Oncology and Dosimetry Committees: EANM procedure guideline for the treatment of liver cancer and liver metastases with intra-arterial radioactive compounds. Eur $\mathrm{J}$ Nucl Med Mol Imaging 38(7), 1393-1406 (2011)

DOI: $10.1007 / \mathrm{s} 00259-011-1812-2$

49. MG. Lam, ML. Goris, AH. lagaru, ES. Mittra, JD. Louie, DY Sze DY: Prognostic utility of $90 Y$ radioembolization dosimetry based on fusion 99mTc-macroaggregated albumin99mTc-sulfur colloid SPECT. J Nucl Med 54(12), 2055-2061 (2013).

DOI: 10.2967/jnumed.113.123257

50. ML. Smits, M. Elschot, MA. van den Bosch, $G H$ van de Maat, $A D$. van het Schip, BA. Zonnenberg, PR. Seevinck, HM. Verkooijen, CJ. Bakker, HW. de Jong, MG. Lam, JF Nijsen: In vivo dosimetry based on SPECT and MR imaging of
$166 \mathrm{Ho}-m i c r o s p h e r e s$ for treatment of liver malignancies. J Nucl Med 54(12), 20932100 (2013)

DOI: 10.2967/jnumed.113.119768

51. S. Lütje, S. Heskamp, AS. Cornelissen, TD. Poeppel, SAMW. van den Broek, S. Rosenbaum-Krumme, A. Bockisch, M. Gotthardt, M. Rijpkema, OC. Boerman: PSMA Ligands for Radionuclide Imaging and Therapy of Prostate Cancer: Clinical Status. Theranostics 5(12), 1388-1401 (2015)

DOI: 10.7150/thno.13348

52. M. Weineisen, M. Schottelius, J. Simecek, RP. Baum, A. Yildiz, S. Beykan, HR. Kulkarni, M. Lassmann, I. Klette, M. Eiber, M. Schwaiger, HJ. Wester: 68Ga- and 177Lu-Labeled PSMA I\&T: Optimization of a PSMA-Targeted Theranostic Concept and First Proof-of-Concept Human Studies. J Nucl Med 56(8), 1169-1176 (2015)

DOI: 10.2967/jnumed.115.158550

53. KL. Chatalic, S. Heskamp, M. Konijnenberg, JD. Molkenboer-Kuenen, GM. Franssen, MC. Clahsen-van Groningen, M. Schottelius, HJ. Wester, WM. van Weerden, OC. Boerman, M. de Jong: Towards Personalized Treatment of Prostate Cancer: PSMA I\&T, a Promising Prostate-Specific Membrane AntigenTargeted Theranostic Agent. Theranostics 6(6), 849-861 (2016)

DOI: $10.7150 /$ thno.14744

Key Words: Radionuclide therapy, Cancer, Imaging, Review

Send correspondence to: Francesca Gallivanone, IBFM-CNR, Segrate, Milan, Italy, Tel: 0039-02-21717511, Fax 0039-02-21717558, E-mail: francesca.gallivanone@ibfm.cnr.it 